\title{
Drying and Hardening Process of Ink dots Investigated by using Digital Holographic Microscopy and Tackiness Measurement
}

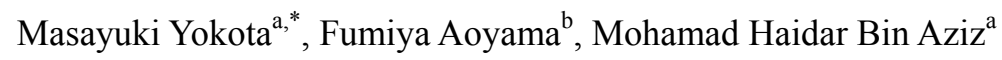 \\ ${ }^{a}$ Faculty of Sci. and Eng., Shimane University, 1060 Nishikawatsu-cho, Matsue-shi, 690-8504, Shimane, Japan) \\ ${ }^{\mathrm{b}}$ Delta Kogyo Co., Ltd., Hiroshima 735-8501, Japan \\ *Corresponding Author: yokota@ecs.shimane-u.ac.jp
}

\begin{abstract}
Drying process of ink dots having diameter of a few hundred micrometer has been investigated with digital holographic microscopy ${ }^{(1-4)}$. In addition, to follow the variation of tackiness of the ink dot in the drying process, a tackiness measurement device (TMD) has been applied to evaluate the change of tackiness of ink. Both the deformation of the ink dot obtained by digital holography and the change of tackiness measured with TMD are compared to investigate the drying process.
\end{abstract}

Keywords: digital holography, microscopy, ink dot, drying process, tackiness measurement.

\section{Introduction}

The knowledge of the drying process of ink has become important in industry for high-quality printing by ink-jet printers. Digital holography has been applied to achieve noncontact assessment of dryness in the drying processes of paint films ${ }^{(5-7)}$. By monitoring the variations occurring in the reconstructed phase for the paint film, it has been possible to evaluate the drying state of paint film up to dry-hard. However, for small ink dot injected by an inkjet printer, digital holography without any magnifying optics has difficulty to evaluate the drying process of such a tiny ink dot due to a lateral resolution.

To investigate the drying process of small ink dot, digital holographic microscopy has been applied to the detection of deformation in the $\operatorname{dot}^{(7)}$. In this paper, we have proposed a digital holographic microscope for evaluating a drying state of a few hundred micrometer sized ink dot injected on an overhead projector (OHP) sheet. In addition, the tackiness of the ink dot is also measured using a TMD with a contact method. By comparing the results of dryness and tackiness of the ink, the drying process of small ink dot have been investigated.

\section{Signal processing}

\subsection{Digital holographic microscope}

To detect the change of wavefront transmitted through the ink dot, off-axis digital holographic microscopy has been adopted ${ }^{(1)}$. The object light $U_{\mathrm{o}}(x, y)$ transmitted through the ink dot is expanded with telecentric imaging system composed of microscope objective lens and a tube lens as seen in Fig. 1. In the system, the object wave at the recording time $t$ becomes ${ }^{(2-4)}$

$$
\begin{aligned}
U_{o}(x, y ; t)=-\frac{k^{2}}{f_{1} f_{2}} & \exp \left\{2 i k \left(f_{1}\right.\right. \\
& \left.\left.+f_{2}\right)\right\}\left\{U\left(\frac{x}{M^{\prime}}, \frac{y}{M^{\prime}} ; t\right) \otimes h(x, y)\right\}
\end{aligned}
$$

where $i$ is the imaginary unit, $M=-f_{2} / f_{1}$ is the magnifying ratio of the lateral size of ink dot, $f_{1}$ and $f_{2}$ are the focal lengths of OL and CL2 in Fig. 1, respectively, $k$ is the wave number, $\otimes$ represents the two-dimensional convolution between the object wave and the point spread function $h$ of the imaging system.

The magnified object wave $U_{\mathrm{ob}}(x, y ; t)$ interferes with the reference light $U_{\mathrm{r}}(x, y ; t)$ reflected from the mirror at the image plane of a digital camera. The intensity of the interference pattern $I_{\text {hol }}(x, y ; t)$, hologram, is expressed as ${ }^{(8)}$ 


$$
\begin{gathered}
I_{h o l}(x, y ; t)=\left|U_{r}\right|^{2}+\left|U_{o b}\right|^{2}+U_{r} U_{o b}^{*}(x, y ; t) \\
+U_{r}^{*} U_{o b}(x, y ; t)
\end{gathered}
$$

where $(x, y)$ denotes the coordinate at the image plane of the camera and * denotes the complex conjugate. To reconstruct the complex amplitude of object wave at the image plane, Fresnel diffraction integral for the hologram is calculated as ${ }^{(8)}$

$$
\begin{gathered}
U_{I}(X, Y ; t)=A \iint R_{D}(x, y) I_{h o l}(x, y ; t) \exp \left[i \frac{\pi}{\lambda z}\{(X\right. \\
\left.\left.-x)^{2}+(Y-y)^{2}\right\}\right]
\end{gathered}
$$

where $z$ is the reconstruction distance, $A=\exp (i k z) /(i \lambda z)$, $(X, Y)$ denotes the coordinate at the image plane and $R_{\mathrm{D}}(x, y)$ is the numerical reference wave to compensate the career component introduced by the off-axis recording. To calculate the equation (3), two-dimensional convolution method is conducted ${ }^{(8)}$.

Thus, the pixel pitch of the reconstructed image becomes the same as that of CMOS digital camera having the $N \times N$ pixels, with a pixel pitch $\Delta x$ and $\Delta y$ in $x$ and $y$ directions. To detect a local variation of the ink dot, the subsequent reconstructed complex amplitudes of object wave is obtained with the interval of $\Delta t$.

The dryness of the ink dot is evaluated by using the standard deviation $\sigma_{\mathrm{t}}$ of the phase difference image between the subsequent reconstructed phases $\Delta \phi_{t}(X, Y)=$ $\arg \left\{U_{\mathrm{I}}(X, Y ; t) U_{\mathrm{I}}^{*}(X, Y ; t+\Delta t)\right\}$. The temporal variation of $\sigma_{\mathrm{t}}$ represents the drying process of ink. To investigate the tiny variation occurring in the ink dot, the unwrapped phase images of $\phi_{t}(X, Y)$ representing the height profile of the ink dot are obtained and the variation of the profile is investigated to see the global trend of the drying process of ink dot.

\subsection{Tackiness measurement}

To evaluate a temporal variation of the tackiness of ink, a tackiness measurement device is used. In the device, a probe having a micro cantilever as a sensing element is touched with the ink surface with manually controlled method. Then, the probe is pulled up to detach it from the ink surface and the force $F$ to detach is measured to evaluate the tackiness of the ink dot. For the purpose, two ink dots are required to separately investigate the temporal variation of the ink due to the contact measurement of tackiness.

\section{Experiment}

Figure 1 shows the experimental setup. The light source is a laser diode (LD) having the wavelength of $\lambda=$ $657.8 \mathrm{~nm}$. As a sample, a commercially available water-based magenta ink for an ink-jet printer was used and applied to an OHP sheet consisting of a plastic sheet and an ink-absorbing film by an ink-jet nozzle. Two ink dots having the diameter of approximately $200 \mu \mathrm{m}$ were made by the nozzle as seen in Fig. 1: the upper dot was used for DH monitoring and the lower one was for TMD. The light from the laser diode transmitting through the upper ink dot was expanded by an objective lens having the magnification of 20 and collimated by a lens (CL2). In the imaging system, the lateral magnification $M=-f_{2} / f_{1}=-7.5$ was achieved.

The transmitted light was recorded with off-axis digital holography in which the off-axis angle for the reference wave was introduced by tilting the mirror (M2). A neutral density filter (ND) was used to adjust the intensity of the reference beam to achieve high contract in the hologram. The hologram was captured by a monochromatic CMOS camera having $2048 \times 2048$ pixels with each pixel size of $5.5 \mu \mathrm{m} \times 5.5 \mu \mathrm{m}$. The image data was sent to a PC via USB3.0 interface.

The hologram recording was conducted with the constant time interval of $\Delta t$ ranging from $0.25 \mathrm{~s}$ to $10.0 \mathrm{~s}$ for a period of 20,000s. Tackiness of the ink dot was also measured manually with the time interval at approximately

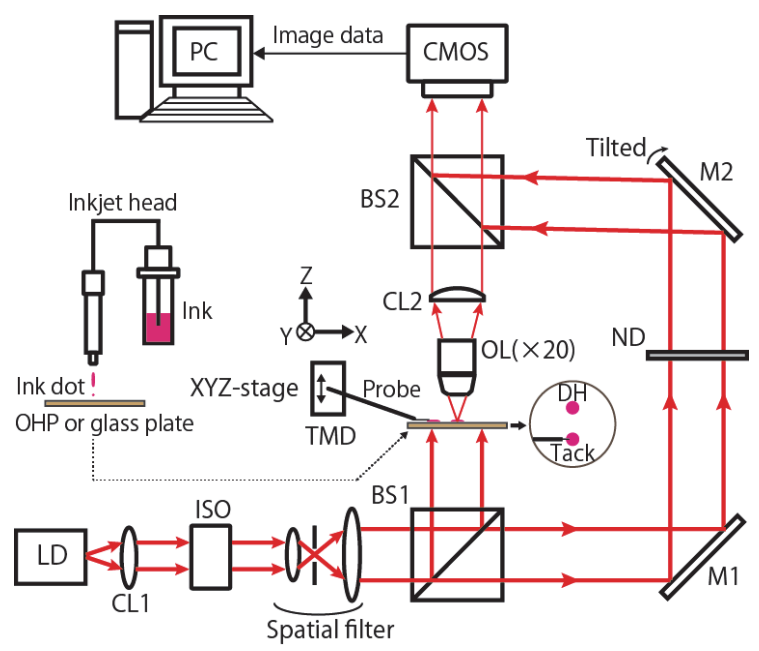

Fig. 1. Experimental setup: ISO; optical isolator, TMD; tackiness measurement device. 
420 s. Tackiness of the ink dot was evaluated by the contacting force of a cantilever sensing head against the ink surface.

\section{Results and Discussions}

Figure 2 shows the variation of both (a) the reconstructed intensity images and (b) phase difference images for the ink dot at various elapsed times. In the phase difference images, the gray scale shows the values of phase difference ranging over $0-2 \pi$ (rad). In Fig. 2(a), no significant change can be seen in the intensity images. On the other hand, the pattern of $\Delta \phi(\Delta t=200 \mathrm{~s})$, which represents the variation of dot occurring in the interval of $\Delta t$ $=200 \mathrm{~s}$, shows some variation before $3000 \mathrm{~s}$. Because the ink dot was so small, no variation of the ink dot could be seen in the phase difference images obtained for shorter interval than $\Delta t=50 \mathrm{~s}$. As seen in Fig. 2(b), the variation in the ink dot seems to end by around $3200 \mathrm{~s}$. To show the global trend of the deformation of the ink dot, the standard deviations $\sigma$ for the phase difference images $\Delta \phi$ obtained for both $\Delta t=100 \mathrm{~s}$ and $200 \mathrm{~s}$ are calculated and shown in Fig. 3 together with the tackiness of the ink dot.

As seen in Fig. 3, the deformation of ink dot represented by the value of $\sigma$ decreases monotonically with time and no significant change is recognized after $3000 \mathrm{~s}$. On the other hand, it the beginning stage of drying process before 2,000 s, the value of tackiness increases and the period corresponds to gelation of the ink. Then, the tackiness of ink rapidly falls at around 10,000 s which corresponds to the start of a solidification of the ink. As seen in Fig. 3, it seems to be difficult to follow the whole drying process of the ink dot by using the variation of $\sigma$.

To follow the deformation of the ink dot after $3000 \mathrm{~s}$,

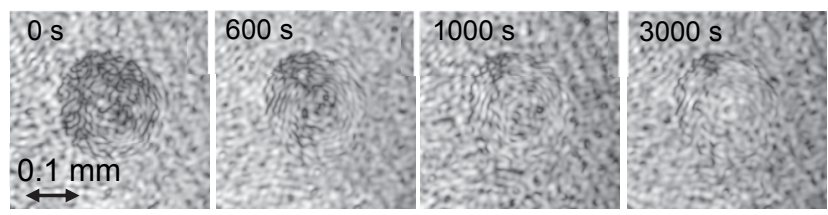

(a) Intensity images.



(b) Phase differnce images $\Delta \phi$ obtained for $\Delta t=200 \mathrm{~s}$.

Fig. 2 Variation of the reconstructed images of the ink dot. the shape of the ink dot has been obtained using unwrapped phase images as shown in Fig. 4 and the cross section of the dot profile at various time lapses is shown in Fig. 5. In Fig. 4 , the areas having phase unwrapping error can be seen in the images of both $0 \mathrm{~s}$ and $600 \mathrm{~s}$. Due to the roughness of OHP film surface, the reconstructed phase images seen in Fig. 2(b) were deformed and that made the phase unwrapping process complicated. However, the global trend of the variation of shape can be analyzed.

In Fig. 5, the height of the ink dot has continued to decrease until 15,000 s. The height of the ink dot decreases at a higher rate before $2,000 \mathrm{~s}$ and the rate of height change reduces after $10,000 \mathrm{~s}$. The trend of height change seems to coincide with the process from gelation to solidification of the ink represented by the tackiness variation in Fig. 3.

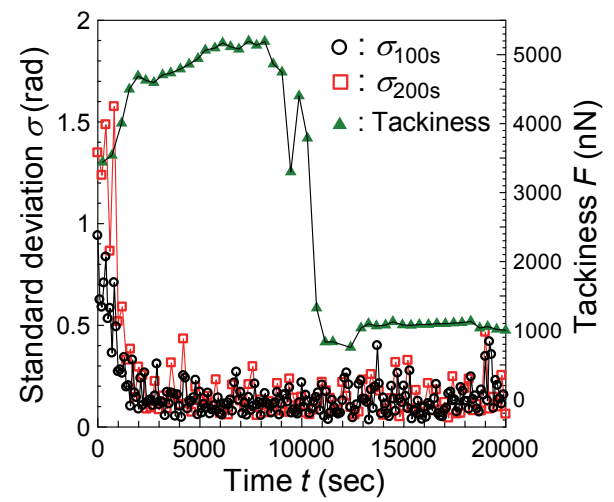

Fig. 3 Standard deviation $\sigma$ and tackiness.

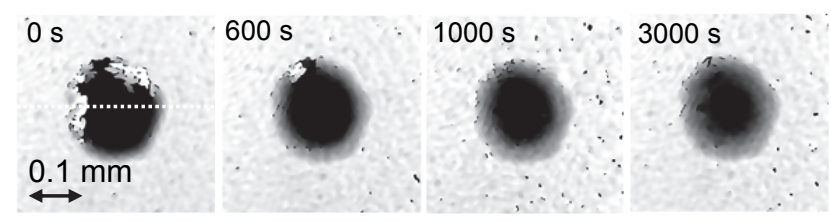

Fig. 4. Unwrapped phase images.

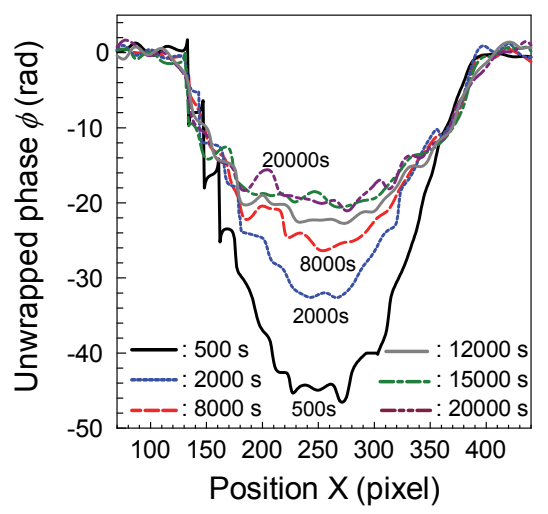

Fig. 5. Cross sectional profile along the white dotted line in Fig.4. 
Figure 6 shows the relationship between the volume estimated from the height profile in Fig. 5 and the variation of tackiness of the ink dot. To estimate the volume of ink dot, the refractive index change due to evaporation of solvent in drying process was ignored and was considered to be constant. In Fig. 6, in the beginning stage of drying process up to $2000 \mathrm{~s}$, the volume of ink dot decreases due to high evaporation of solvent. However, after $2000 \mathrm{~s}$, the rate rapidly decreases and becomes almost constant. The variation of volume continues after 20000 s. Thus, the drying process of ink dot continued even though the tackiness change seems to shrink. The volume change of ink dot may come from the evaporation of solvent in the dot. The hardening process of ink dot had continued after the experiment. The process might continue more than two days in this case.

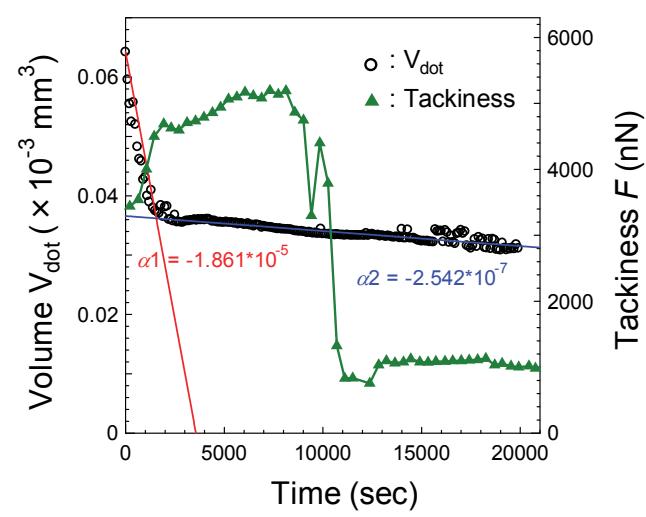

Fig. 6. Variation of the volume and the tackiness of the ink dot on OHP film.

\section{Conclusions}

Digital holographic microscopy has been applied to evaluate the drying process of a few hundred micrometer sized ink dot. By monitoring the temporal variation of the shape of ink dot, the drying process can be followed up to the solidification of the ink. Tackiness measurement has also performed in a simultaneous manner. The combination method using both digital holographic microscopy and tackiness measurement is useful for the investigation of whole drying process of small ink dot ranging from gelation to solidification.

\section{Acknowledgment}

This work was partially supported by MEXT KAKENHI Grant Number 15K06109.

\section{References}

(1) Myung. K. Kim : "Principles and techniques of digital holographic microscopy", SPIE Reviews, Vol. 1, pp. 018005-1-018005-50, 2010

(2) Ana Doblas, Emilio Sanchez-Ortiga, Manuel Martinez-Corral, Genaro Saavedra, Pedro Andres, and Jorge Garcia-Sucerquia : "Shift-variant digital holographic microscopy: inaccuracies in quantitative phase imaging", Optics Letters, Vol. 38, No. 8, pp. 1352-1354, 2013

(3) Yun Liu, Zhao Wang, Jiansu Li, Jianmin Gao, and Junhui Huang : "Total aberrations compensation for misalignment of telecentric arrangement in digital holographic microscopy", Optical Engineering, Vo. 53, No. 11, pp. 112307-1-112307-8, 2014

(4) Emilio Sanchez-Ortiga, Ana Doblas, Genaro Saavedra, Manuel Martinez-Corral, and Jorge Garcia-Sucerquia : "Off-axis holographic microscopy: practical design parameters for operating at diffraction limit", Applied Optics, Vol. 53, No. 10, pp. 2058-2066, 2014

(5) Masayuki Yokota, Toru Adachi and Ichirou Yamaguchi : "Monitoring and evaluation of drying of paint by using phase-shifting digital holography", Optical Engineering, Vol. 49, pp. 015801-1-015801-4, 2010

(6) Masayuki Yokota, Tomoaki Kawakami and Ichirou Yamaguchi : "Drying process in a solvent-based paint analyzed by phase-shifting digital holography and an estimation of time for tack free", Applied Optics, Vol. 50, No. 30, pp. 5834-5841, 2011

(7) Masayuki Yokota and Yoshiki Kimoto : "Combination method of digital holography and gravimetric measurement for assessment of a paint drying process", Optical Engineering, Vol. 52, No. 1, pp. 015801-1-015801-10, 2013

(8) Ulf Schnars and Werner Jueptner : "Digital Holography”, Springer, New York, 2005 